\title{
Should Contralateral Nodules Be an Indication of Total or Completion Thyroidectomy for Patients With Unilateral Papillary Thyroid Carcinoma?
}

OPEN ACCESS

Edited by:

Paolo Miccoli,

University of Pisa, Italy

Reviewed by:

Leonardo Rossi, University of Pisa, Italy

Francesca Ragusa, University of Pisa, Italy

*Correspondence:

Jifeng Liu

729122921@qq.com

Specialty section:

This article was submitted to

Thyroid Endocrinology,

a section of the journal

Frontiers in Endocrinology

Received: 11 June 2021

Accepted: 27 July 2021

Published: 09 August 2021

Citation:

Ma T, Wang H, Liu J, Zou J and Liu S

(2021) Should Contralateral Nodules Be an Indication of Total

or Completion Thyroidectomy

for Patients With Unilateral

Papillary Thyroid Carcinoma?

Front. Endocrinol. 12:723631.

doi: 10.3389/fendo.2021.723631
Tengfei Ma, Haiyang Wang, Jifeng Liu*, Jian Zou and Shixi Liu

Department of Otolaryngology Head \& Neck Surgery, West China Hospital, Sichuan University, Chengdu, China

Objective: To determine whether papillary thyroid carcinoma (PTC) patients with benign or nonsuspicious nodules in the contralateral lobe have a higher rate of recurrence or worse survival after lobectomy compared to those without nodules in the contralateral lobe.

Methods: Adult patients who underwent lobectomy and were diagnosed with unilateral PTC (2013-2015), were identified from an institutional database. Patients who previously had cytologically benign nodules or nonsuspicious nodules in the contralateral lobe comprised the contralateral nodule (CN) group. Patients who did not have nodules in the contralateral lobe comprised the unilateral nodule (UN) group.

Results: 370 patients were included: 242 in the UN group and 128 in the CN group. After a median follow-up of 62 months (range, 16-85 months), recurrence was confirmed in 4.1\% patients in the UN group and 5.5\% patients in the CN group $(p=0.559)$. Clinical contralateral lobe PTC was detected in 2.9\% (7/242) of patients from the UN group and 3.9\% (5/128) of patients from the $\mathrm{CN}$ group $(p=0.601)$. The 5 -year contralateral lobe recurrence-free survival (RFS) rates were $96.8 \%$ in the UN group and $97.4 \%$ in the CN group ( $p=0.396$ ). The 5-year loco-regional RFS rates were $98.4 \%$ in the UN group and $97.8 \%$ in the CN group $(p=0.690)$. The 5 -year disease-specific survival rates were both $100 \%$.

Conclusion: PTC patients with benign or nonsuspicious $\mathrm{CN}$ s have similar recurrence and survival rates after lobectomy compared to those without CNs. CNs alone should not be an indication for total or completion thyroidectomy.

Keywords: papillary thyroid carcinoma, contralateral nodules, lobectomy, total thyroidectomy, completion thyroidectomy, recurrence, clinical contralateral PTC 


\section{INTRODUCTION}

The prevalence of thyroid nodules among asymptomatic persons is about $19-70 \%(1-3)$. About $8-16 \%$ of thyroid nodules are malignant (1), of which papillary thyroid cancers (PTCs) are the most common $(4,5)$. In the past few decades, total thyroidectomy has been the main treatment for PTC. However, in recent years, many experts have called for less aggressive treatment of PTC, as studies have shown that total thyroidectomy does not provide a better clinical outcome than unilateral lobectomy for patients with low or intermediate risk PTC (6-9). In agreement with this conservative trend, most clinical guidelines currently recommend lobectomy as a valid treatment option for patients with a tumor $<4 \mathrm{~cm}$, without gross extrathyroidal extension (ETE), and non-metastatic disease (10-12).

The incidence of thyroid cancer, mainly PTC, has increased sharply worldwide over the past 30 to 40 years $(13,14)$. Given the striking prevalence of thyroid nodules and the increasing incidence of PTC, it is common to detect bilateral thyroid nodules by ultrasonography (US) in patients with pathologically confirmed PTC in one lobe (15).

For patients diagnosed with unilateral PTC, nodules in the contralateral thyroid lobe have been considered a factor influencing the extent of surgery. Indeed, according to the 2015 American Thyroid Association (ATA) guidelines and the National Comprehensive Cancer Network guidelines (version 2.2020), the presence of contralateral thyroid nodules in PTC patients may be criteria for recommending total or completion thyroidectomy to better address suspicions of bilateral disease and facilitate follow-up $(11,12)$. Several studies may favor these recommendations, since up to $48 \%$ of patients diagnosed with unilateral PTC have PTCs in the contralateral lobe $(16,17)$. However, these studies have focused on patients who underwent total thyroidectomy or completion thyroidectomy. The natural follow-up outcomes of patients with unilateral PTC and contralateral nodules (CNs) after lobectomy have rarely been studied. A recent study by Ritter et al. (15) showed that in PTC patients with benign or nonsuspicious CNs under close followup, the rate of contralateral lobe PTC after lobectomy was only $5 \%$.

Accordingly, we hypothesize that lobectomy is an appropriate treatment for low-risk PTC patients (tumor $<4 \mathrm{~cm}$, non-gross ETE, and non-metastatic disease) with contralateral thyroid lobe nodules. Therefore, we determined whether PTC patients with nonsuspicious $\mathrm{CNs}$ have worse outcomes after lobectomy.

\section{MATERIALS AND METHODS}

\section{Study Cohort}

This study included adult patients ( $\geq 18$ years), who underwent lobectomy and were diagnosed with unilateral PTC by postoperative pathology from January 2013 to December 2015 at West China Hospital of Sichuan University. The extent of surgery was at the discretion of the treating surgeon with consideration for patient preference. In our hospital, ipsilateral prophylactic central-compartment neck dissection was performed for all patients with PTC. Exclusion criteria were: primary tumor size $\geq 4 \mathrm{~cm}$, gross ETE, aggressive histology (tall cell, columnar cell, and hobnail variants) and/or vascular invasion, clinical cervical lymph node metastasis, and evidence of distant metastasis. Included patients had at least 12 months follow-up after surgery. Patients were categorized into two groups. Patients who did not have nodules in the contralateral lobe comprised the unilateral nodule (UN) group, and patients who previously had cytologically benign nodules defined by fine needle aspiration or nonsuspicious nodules by US in contralateral lobe comprised the $\mathrm{CN}$ group. The risk of malignancy of the contralateral thyroid nodules was estimated according to the sonographic pattern classification proposed by the 2015 ATA guidelines (11). Nonsuspicious nodules were defined as nodules without ATA high-risk sonographic features (microcalcifications, irregular margins, taller than wide shape, rim calcifications with small extrusive soft tissue component, and evidence of ETE). All patients were followed with thyroid and neck US every 3 months in the first year after initial surgery; if disease-free, they were followed up every 6 months in the second year and then annually since the third year. Disease-free was defined as the absence of suspicious findings on the neck US. During the follow-up period, fine needle aspiration (FNA) was generally recommended for patients with suspicious findings on neck US. Referring to previous literature, recurrence after unilateral thyroid lobectomy was classified as recurrence in the contralateral lobe and loco-regional (ipsilateral thyroid bed or cervical lymph nodes) recurrence (18). Patients with confirmed or suspicious contralateral lobe recurrence were recommended to receive completion thyroidectomy and central-compartment neck dissection. Selective neck dissection was performed for those with confirmed or suspicious locoregional recurrence.

The following data were retrospectively reviewed and analyzed: age at diagnosis, gender, tumor size, microscopic ETE condition, and tumor stage. Tumor stage was classified according to the American Joint Committee on Cancer (AJCC) 8th Edition TNM staging system (19). The data of postoperative complications including voice impairment (hoarseness, voice fatigue, low-pitch voice), dysphagia, and hypocalcemia was recorded. The information of voice impairment and dysphagia were obtained through physicians' active inquiry. Hypocalcemia was defined as serum calcium below the lower limit of the normal range at our institution $(2.11-2.52 \mathrm{mmol} / \mathrm{l})$ after unilateral thyroidectomy within $48 \mathrm{~h}$ or during the follow-up period and/ or the need for vitamin $\mathrm{D}$ and calcium supplements. Complications lasting within and beyond 6 months were defined as transient and persistent, respectively. Contralateral lobe recurrence-free survival (RFS), loco-regional RFS, diseasespecific survival (DSS), and overall survival (OS) were calculated in months.

\section{Statistical Analyses}

Statistical analyses were conducted using SPSS, v.25.0 (IBM Corp., Armonk, NY, USA). Categorical variables were analyzed 
by the $\chi^{2}$ test and are presented as numbers with percentages. Continuous variables were analyzed by the Student's t-test and are presented as the mean with standard deviation, or median and range, when appropriate. The Kaplan-Meier method was used to analyze the survival outcomes and calculate the 5-year survival rates. $p<0.05$ was considered statistically significant.

\section{RESULTS}

\section{Comparisons of Patients and Tumor Characteristics}

Of the 409 patients initially included, 39 patients with a followup of less than 12 months were excluded. Finally, 370 patients diagnosed with unilateral PTC after undergoing lobectomy and ipsilateral central-compartment neck dissection met the inclusion criteria for this retrospective study. Among them, 128 patients $(128 / 370,34.6 \%)$ belonged to the $\mathrm{CN}$ group. In the cohort, the median follow-up time was 62 months, ranging from 16 to 85 months. Excluding two patients who died from other disease at 16 and 18 months after initial surgery, the shortest follow-up time was 46 months. The median follow-up time for the UN and $\mathrm{CN}$ groups was 64 months (ranging from 16 to 85 ) and 57 months (ranging from 18 to 83 ), respectively ( $p=$ 0.609). The median age at diagnosis for all patients was 38 (ranging from 18 to 78 ) years old, and 314 (84.9\%) patients were younger than 55 years old. Most patients were female (76.5\%). Mean tumor size was $1.40 \pm 0.96 \mathrm{~cm}$ and 154 patients (41.6\%) had a tumor $\leq 1 \mathrm{~cm}$. Microscopic ETE was observed in 23 patients $(6.2 \%)$. Histological patterns of PTC included classical $(82.7 \%)$ and follicular (17.3\%) variants. All patients from both groups were classified as AJCC Stage I. There were no significant differences in gender $(p=0.189)$, PTC subtype $(p=0.232)$, or presence of microscopic ETE ( $p=0.665)$ between the two groups. Patients in the $\mathrm{CN}$ group were older $(p=0.001)$ and had a larger tumor $(p<0.001)$ than those in the UN group. The proportion of patients older than 55 years old in the $\mathrm{CN}$ group was higher than that in the UN group $(p=0.02)$. Patients in the $\mathrm{CN}$ group had a higher frequency of tumors $>1 \mathrm{~cm}(p=0.023)$. Table 1 summarizes the patient and tumor characteristics.

\section{CNs}

The mean size of the contralateral lobe nodules detected by US in the CN group was $0.52 \pm 0.32 \mathrm{~cm}$, and $7.8 \%(10 / 128)$ were $>1 \mathrm{~cm}$. Six patients underwent FNA biopsy of the contralateral lobe nodules, and the results were benign (Bethesda II). The proportions of very low-suspicion, low-suspicion, and intermediate-suspicion nodules were $11.7 \%, 20.3 \%$, and $62.5 \%$ respectively (Table 2 ). No patient had a high-suspicion sonographic pattern. There were seven patients who could not be classified due to the lack of echo intensity description in the US report.

\section{Postoperative Complications}

Among all of the patients, 10.5\% (39 patients) had transient voice impairment and 3.8\% (14 patients) suffered from persistent voice impairment. The most common voice impairment symptom was hoarseness (12.2\% transient and $0 \%$ persistent), and the most common persistent voice impairment symptom was low-pitch voice $(2.2 \%$ persistent). In addition, some patients developed hypocalcemia (2.4\% transient and $0 \%$ persistent) and dysphagia ( $1.6 \%$ transient and $0.8 \%$ persistent). There were no significant differences in the frequency of complications between the two groups (Table 3 ).

\section{Comparison of Recurrence and Survival Outcomes}

After a median follow-up period of 62 months, $4.1 \%$ (10/242) of the patients in the UN group and $5.5 \%(7 / 128)$ of the patients in the $\mathrm{CN}$ underwent reoperation $(p=0.559)$. All of them had tumor recurrence proved by pathological analysis. Table 4 shows the characteristics of these patients. One patient in the CN group

TABLE 1 | Comparison of patient and tumor characteristics between the UN and CN nodule groups.

\begin{tabular}{|c|c|c|c|c|}
\hline & Total $(n=370)$ & UN group $(n=242)$ & $C N$ group $(n=128)$ & $p$-value \\
\hline \multicolumn{5}{|l|}{ Age } \\
\hline Mean age & $39.8 \pm 12.8 y$ & $38 \pm 12.3 y$ & $43 \pm 13.1 y$ & 0.001 \\
\hline$<55$ & 314 (84.9\%) & 213 (88\%) & 101 (78.9\%) & 0.020 \\
\hline$\geq 55$ & $56(15.1 \%)$ & $29(12 \%)$ & 27 (21.1\%) & \\
\hline \multicolumn{5}{|l|}{ Gender } \\
\hline Female & $283(76.5 \%)$ & 180 (74.4\%) & 103 (80.5\%) & 0.189 \\
\hline Male & $87(23.5 \%)$ & $62(25.6 \%)$ & 25 (19.5\%) & \\
\hline \multicolumn{5}{|l|}{ Tumor size } \\
\hline Mean size & $1.40 \pm 0.96 \mathrm{~cm}$ & $1.26 \pm 0.84 \mathrm{~cm}$ & $1.67 \pm 1.12 \mathrm{~cm}$ & $<0.001$ \\
\hline$\leq 1 \mathrm{~cm}$ & 154 (41.6\%) & 111 (45.9\%) & 43 (33.6\%) & 0.023 \\
\hline $1-4 \mathrm{~cm}$ & $216(58.4 \%)$ & $131(54.1 \%)$ & 85 (66.4\%) & \\
\hline \multicolumn{5}{|l|}{ PTC subtype } \\
\hline Classical variant & 306 (82.7\%) & $196(81 \%)$ & 110 (85.9\%) & 0.232 \\
\hline Follicular variant & $64(17.3 \%)$ & 46 (19\%) & $18(14.1 \%)$ & \\
\hline \multicolumn{5}{|l|}{ Microscopic ETE } \\
\hline Yes & $23(6.2 \%)$ & $16(6.6 \%)$ & $7(5.5 \%)$ & 0.665 \\
\hline No & 337 (93.8\%) & 226 (93.4\%) & 121 (94.5\%) & \\
\hline Median follow-up time & $62(16,85) \mathrm{m}$ & $64(16,85) \mathrm{m}$ & $57(18,83) \mathrm{m}$ & 0.609 \\
\hline
\end{tabular}

UN, unilateral nodule; CN, contralateral nodule; PTC, papillary thyroid carcinoma; ETE, extrathyroidal extension. 
TABLE 2 | ATA sonographic pattern of CNs.

ATA sonographic pattern

$128(\%)$

Very low-suspicion

Low-suspicion

Intermediate-suspicion

High-suspicion

Unknown respectively (Figure 1). The rates of loco-regional recurrence were $1.6 \%(2 / 242)$ in the CN group and $2.5 \%(6 / 128)$ in the UN group $(p=0.564)$. The 5-year loco-regional RFS rates in the $\mathrm{CN}$ and UN groups were $98.4 \%$ and. $97.8 \%$, respectively $(p=0.690$;

Figure 2). There were four non-disease-specific deaths: two patients in the UN group died from an unknown cause and breast cancer, respectively, and two patients in the CN group died from lung cancer and ovarian cancer, respectively. The 5year DSS rates were both $100 \%$, and the 5-year OS rates were $99.2 \%$ in the $\mathrm{CN}$ group and $98.4 \%$ in the UN group $(p=0.517)$.

\section{DISCUSSION}

Despite the sharp increasing incidence in PTC, the mortality rate has remained unchanged and the 20-year survival rate of these patients is close to $95 \%$ (20). It is widely recognized that the increasing incidence is largely due to overdiagnosis caused by the detection of subclinical cancers that would never cause harm (21),

TABLE 3 | Postoperative complications of patients in the two groups.

\begin{tabular}{|c|c|c|c|c|}
\hline & Total $(n=370)$ & UN group $(n=242)$ & CN group $(n=128)$ & $p$-value \\
\hline Voice impairment & & & & 0.337 \\
\hline No & 317 (85.7\%) & 207 (85.8\%) & 110 (85.9\%) & \\
\hline Transient & 39 (10.5\%) & $28(11.6 \%)$ & 11 (8.6\%) & \\
\hline Persistent & $14(3.8 \%)$ & 7 (2.9\%) & 7 (5.5\%) & \\
\hline Dysphagia & & & & 0.199 \\
\hline No & 361 (97.6\%) & 234 (96.7\%) & 127 (99.2\%) & \\
\hline Transient & $6(1.6 \%)$ & $6(2.5 \%)$ & $0(0 \%)$ & \\
\hline Persistent & $3(0.8 \%)$ & $2(0.8 \%)$ & $1(0.8 \%)$ & \\
\hline Hypocalcemia & & & & 0.936 \\
\hline No & 361 (97.6\%) & 236 (97.5\%) & 125 (97.7\%) & \\
\hline Transient & $9(2.4 \%)$ & $6(2.5 \%)$ & $3(2.3 \%)$ & \\
\hline Persistent & $0(0 \%)$ & $0(0 \%)$ & $0(0 \%)$ & \\
\hline
\end{tabular}

UN, unilateral nodule; $C N$, contralateral nodule.

TABLE 4 | Cases of patients with recurrence.

\begin{tabular}{|c|c|c|c|c|c|}
\hline No. & Group & Site of recurrence & Time to recurrence $(\mathrm{m})$ & Management of recurrence & Final outcome \\
\hline 1 & UN & Contralateral lobe & 49 & $\mathrm{CT}+\mathrm{CCND}$ & NED \\
\hline 2 & UN & Contralateral lobe & 14 & $\mathrm{CT}+\mathrm{CCND}$ & NED \\
\hline 3 & UN & Ipsilateral level VI CLN & 10 & SND & NED \\
\hline 4 & UN & Contralateral lobe & 20 & $\mathrm{CT}+\mathrm{CCND}$ & NED \\
\hline 5 & UN & Ipsilateral level II-V CLN & 71 & SND & NED \\
\hline 6 & UN & Contralateral lobe, Ipsilateral thyroid bed, bilateral level II-VI CLN & 59 & $\mathrm{CT}+\mathrm{SND}$ & NED \\
\hline 7 & UN & Contralateral lobe, contralateral level VI CLN & 39 & $\mathrm{CT}+\mathrm{CCND}$ & NED \\
\hline 8 & UN & Contralateral lobe, contralateral level VI CLN & 38 & $\mathrm{CT}+\mathrm{CCND}$ & NED \\
\hline 9 & UN & Ipsilateral level II, III CLN & 13 & SND & NED \\
\hline 10 & UN & Contralateral lobe & 68 & $\mathrm{CT}+\mathrm{CCND}$ & NED \\
\hline 11 & $\mathrm{CN}$ & Contralateral lobe & 6 & $\mathrm{CT}+\mathrm{CCND}$ & NED \\
\hline 12 & $\mathrm{CN}$ & Ipsilateral level II-VI CLN & 10 & SND & NED \\
\hline 13 & $\mathrm{CN}$ & Contralateral lobe & 10 & $\mathrm{CT}+\mathrm{CCND}$ & NED \\
\hline 14 & $\mathrm{CN}$ & Ipsilateral level II, III, VI CLN & 50 & $\mathrm{CT}+\mathrm{SND}$ & NED \\
\hline 15 & $\mathrm{CN}$ & Contralateral lobe & 40 & $\mathrm{CT}+\mathrm{CCND}$ & NED \\
\hline 16 & $\mathrm{CN}$ & Contralateral lobe & 63 & $\mathrm{CT}+\mathrm{CCND}$ & NED \\
\hline $17^{\star}$ & $\mathrm{CN}$ & Contralateral lobe & 42 & $\mathrm{CT}+\mathrm{CCND}$ & NED \\
\hline
\end{tabular}

UN, unilateral nodule; CN, contralateral nodule; CT, completion thyroidectomy; CCND, central-compartment neck dissection; SND, selective neck dissection; NED, no evidence of disease. *Patient No.17 underwent another selective neck dissection 20 months after the second surgery, and there was no evidence of recurrence during the 17-month follow-up period after the third surgery. 


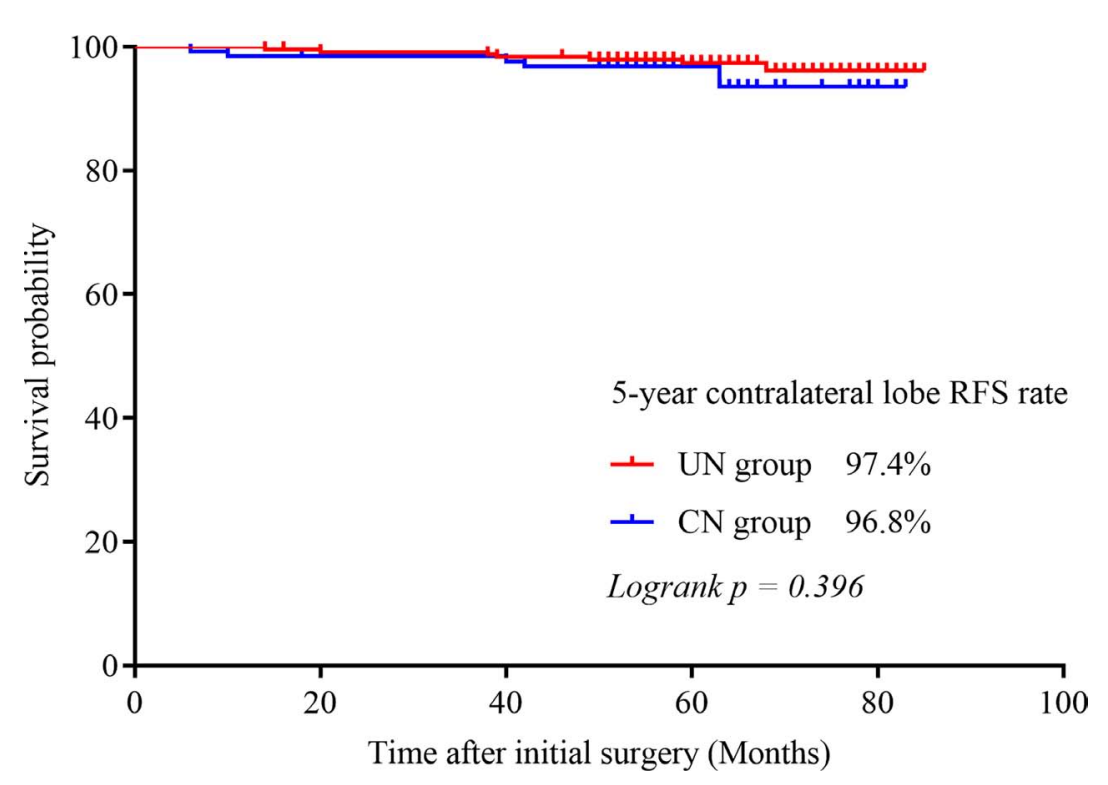

FIGURE 1 | Kaplan-Meier survival curve illustrating the 5-year contralateral lobe RFS rate in both groups.

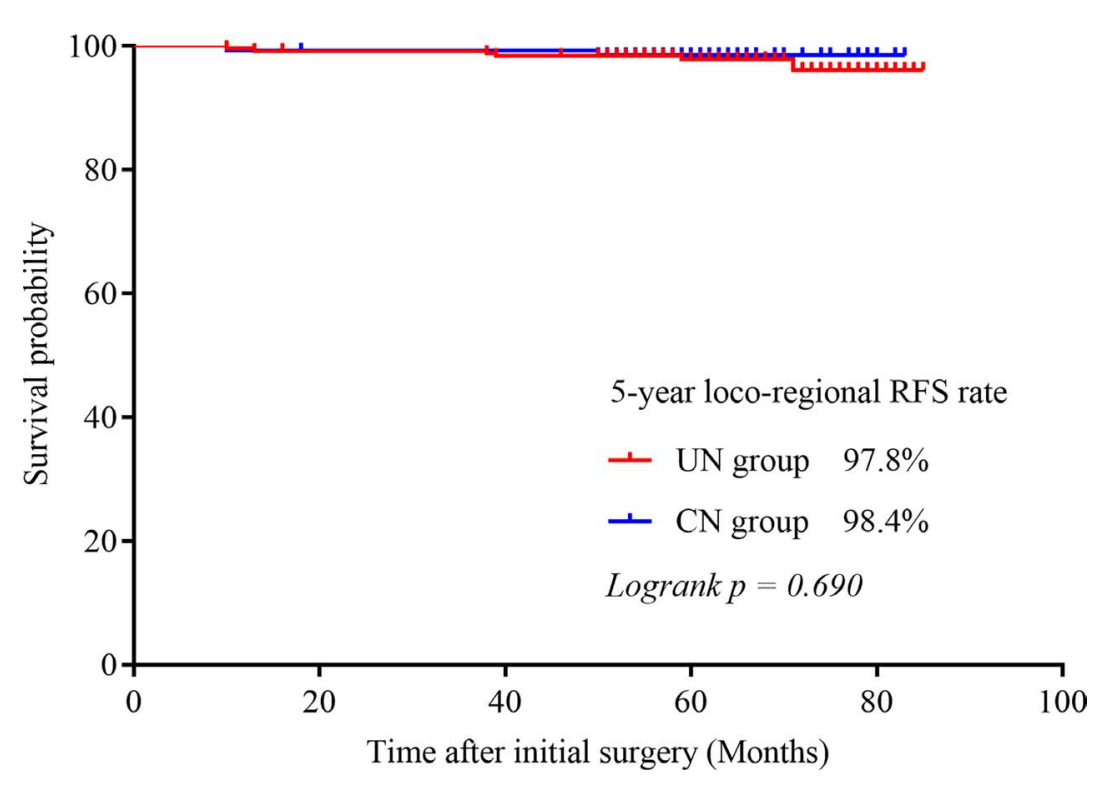

FIGURE 2 | Kaplan-Meier survival curves illustrating the 5-year loco-regional RFS rate in the two groups.

which certainly leads to overtreatment (22). Therefore, clinical guidelines are calling for low-intensity treatment of PTC $(11,12)$. Previously, total thyroidectomy was the main treatment for PTC, while unilateral thyroid lobectomy was only performed for patients with microcarcinoma (tumor $<1 \mathrm{~cm}$ ) (23). However, in the past decade, studies have confirmed that patients with low- or intermediate-risk PTC who undergo total thyroidectomy have similar recurrence and survival rates as those who undergo unilateral thyroid lobectomy $(6-9,24)$. At present, most clinical guidelines consider unilateral thyroid lobectomy a valid treatment option for patients with tumor $<4 \mathrm{~cm}$, non-gross ETE, and nonmetastatic disease (10-12).

One of the benefits supporting this conservative trend is that patients after unilateral thyroid lobectomy have a lower incidence of postoperative adverse events. In a retrospective study of 62,722 patients, Hauch et al. (25) found that the overall rate of complications after unilateral thyroid lobectomy was $10.8 \%$, which was significantly lower than that after total thyroidectomy $(20.4 \%, P<0.0001)$. The authors also found that the rate of complications after total thyroidectomy decreased 
with the surgeon's experience, but even for high-volume surgeons (those performing $>99$ thyroidectomies/year), this rate $(14.5 \%)$ was still higher than that after unilateral thyroid lobectomy $(7.6 \%, p<0.0001)(25)$.

Moreover, many studies have shown that thyroid lobectomy is more effective than total thyroidectomy in reducing various postoperative complications such as hypocalcemia and recurrent laryngeal nerve injury (26-28). The incidence of persistent complications after unilateral lobectomy in our study was also much lower than total thyroidectomy reported in previous articles. The rate of persistent voice impairment, hypocalcemia, and dysphagia after unilateral thyroid lobectomy in our study were $3.8 \%, 0 \%$, and $0.8 \%$, respectively. While in different studies, the incidence of these three persistent complications after total thyroidectomy can be up to $31 \%(29,30), 6.8 \%(31-33)$, and $20 \%$ $(34,35)$, respectively. More importantly, thyroid lobectomy does not cause the rare but extremely serious postoperative complication of bilateral recurrent laryngeal nerve paralysis, which generally results in severe dyspnea requiring tracheostomy (36).

Furthermore, patients undergoing total thyroidectomy must receive lifelong thyroid hormone replacement therapy, while thyroid lobectomy gives a chance to avoid this tedious daily task and its potential adverse effects (37). In addition to replacing endogenous thyroid hormone, the other purpose of hormone replacement therapy after thyroidectomy is to indirectly prevent recurrence or progression of thyroid cancer through negative feedback on pituitary thyroid-stimulating hormone (TSH) secretion (38). However, due to the lack of evidence that lowrisk patients after lobectomy can benefit from postoperative TSH suppression, there is no consensus on postoperative hormone therapy for these patients (11). The 2015 ATA guidelines recommend that low-risk patients who undergo unilateral lobectomy maintain TSH level in the low to moderate normal range $(0.5-2 \mathrm{mU} / \mathrm{L})$, and patients with $\mathrm{TSH}$ in this range do not need thyroid hormone therapy (11). Kim et al. (39) showed that for low-risk PTC patients undergoing unilateral lobectomy, 34\% did not need hormone replacement therapy and the remaining $66 \%$ used levothyroxine to maintain normal (rather than suppressed) TSH levels (0.86-4.69 $\mathrm{mcIU} / \mathrm{mL})$; after 5-year follow-up, recurrence was only detected in $1 \%$ of the patients. Similarly, Lee et al. (40) found that even for those who received TSH suppression therapy, $53.2 \%$ successfully stopped using levothyroxine when they maintained euthyroid status and only $0.3 \%$ of the patients had recurrence.

As discussed above, clinical guidelines are calling for more conservative extent of thyroidectomy for PTC, which raises the question of whether contralateral lobe nodules should still be an indication of total or completion thyroidectomy. Evidence supporting total or complete thyroidectomy is the probable high risk of contralateral malignancy in patients with unilateral PTC. Ibrahim et al. (16) studied 97 patients who were diagnosed with PTC after initial lobectomy and subsequently received completion thyroidectomy; the results showed that 48\% (47/97) had contralateral lobe PTC. However, Ibrahim et al. (16) did not take into account the US features of the residual contralateral lobe.
Wu et al. (17) analyzed the pathology of 347 cases of papillary thyroid microcarcinoma with ultrasonically benign-like CNs after total thyroidectomy, and found that $28.9 \%$ (100/347) had contralateral PTC. Notably, patients in these two studies underwent total or completion thyroidectomy, while those who received lobectomy alone were not considered; this selection bias could lead to a higher probability of finding a contralateral PTC. Recently, Ritter et al. (15) studied 112 PTC patients with solid contralateral benign or nonsuspicious thyroid nodules who underwent thyroid lobectomy alone; they found that after a median follow-up of 6 years, 11\% (12/112) patients received completion thyroidectomy, and only 5\% (6/112) were pathologically proven to have a PTC in the contralateral lobe.

In our study, we compared the outcomes between PTC patients with or without contralateral lobe nodules after unilateral thyroid lobectomy. Firstly, after a median follow-up period of 62 months, for patients with benign or nonsuspicious $\mathrm{CN}$, the rate of clinical contralateral lobe PTC was only $3.9 \%$ (5/ $128)$ and the rate of loco-reginal recurrence was $1.6 \%(2 / 242)$. Second, in our study, there was no significant difference between patients with $\mathrm{CN}$ and patients without $\mathrm{CN}$ in terms of contralateral recurrence and loco-regional recurrence rate (5year contralateral lobe RFS rates were $96.8 \%$ and $97.4 \%$, respectively, $p=0.396$; 5 -year loco-regional RFS rates were 98.4\% and 97.8\%, $p=0.690$ ); and the DSS and OS rates were similar between the two groups (5-year OS rates were $98.4 \%$ and $99.2 \%$ respectively, $p=0.517$; 5 -year DSS rate was both $100 \%$ ).

In addition, a recent study by Sun et al. (41) showed that patients without nodules in the contralateral thyroid lobe at the time of initial lobectomy had a high probability, up to $41.9 \%$, of developing nodules in the contralateral lobe, but only $6.5 \%$ of these nodules were proven to be clinically malignant. The rest of ultrasonically nonsuspicious new nodules achieved favorable clinical outcomes under ultrasound surveillance (41).

Consistent with the study from Ritter et al. (15) and Sun et al. (41), we proved that low-risk PTC patients with contralateral benign or nonsuspicious nodules have a low risk of developing clinical contralateral PTC. Our results further suggest that benign or nonsuspicious nodules in the contralateral lobe do not increase the risk of recurrence and disease-specific death. Moreover, completion thyroidectomy and (or) selective neck dissection remain timely and effective for recurrence found during follow-up. Therefore, though several studies have reported that PTC patients with $\mathrm{CN}$ have a high probability of contralateral PTC $(16,17)$, this favorable result suggests that if the contralateral lobe is removed without evidence of malignancy, it may lead to the overdiagnosis of contralateral subclinical PTC, which will certainly result in overtreatment to some extent.

Additional support for the aggressive surgical treatment is that the 2015 ATA guidelines recommend that PTC patients with contralateral thyroid nodules undergo total or completion thyroidectomy in order to carry out the postoperative radioiodine (RAI) therapy plan (11). However, several recent studies demonstrated that RAI therapy should not be routinely administered to patients with low-risk PTC. Ibrahimpasic et al. (42) 
reported that, after a follow-up time of 62 months, low-risk PTC patients managed without RAI had a similar RFS to those managed with RAI (96\% vs. $100 \%, p=0.337$ ) after total thyroidectomy. According to a large population study of 276,558 patients, Orosco et al. (43) found that low-risk patients benefited the least from RAI treatment; by contrast, iodine therapy might even increase diseasespecific mortality in low-risk T1a patients.

Although our study confirmed the benefits and safety of unilateral thyroid lobectomy for low-risk PTC patients with benign and nonsuspicious nodules, there is no doubt that postoperative lifelong follow-up is a challenge for both patients and physicians. In particular, some patients prefer total thyroidectomy as the initial treatment in order to avoid completion thyroidectomy for recurrence in contralateral thyroid lobe. Surgeons should take the patient's preference and compliance of the postoperative follow-up into account when considering the extent of the thyroidectomy.

This study had some limitations. First, it was a retrospective and single-center study. Second, 39 patients were excluded due to insufficient postoperative follow-up data, which may have led to selection bias. Third, since the 30 -year postoperative recurrence rate of differentiated thyroid carcinoma is up to $30 \%$ and $66 \%$ of recurrences occur in the first 10 years after operation (44), the follow-up period of our study may not be long enough to reveal the differences in recurrence rates between the two groups.

\section{CONCLUSION}

PTC patients with benign or nonsuspicious $\mathrm{CNs}$ have similar recurrence and survival rates after unilateral lobectomy compared to those without CNs. Contralateral thyroid nodules alone should not be an indication for total or completion thyroidectomy.

\section{REFERENCES}

1. Burman KD, Wartofsky L. Clinical Practice. Thyroid Nodules. N Engl J Med (2015) 373(24):2347-56. doi: 10.1056/NEJMcp1415786

2. Walsh JP. Managing Thyroid Disease in General Practice. Med J Aust (2016) 205(4):179-84. doi: 10.5694/mja16.00545

3. Hegedüs L. Clinical Practice. The Thyroid Nodule. N Engl J Med (2004) 351 (17):1764-71. doi: 10.1056/NEJMcp031436

4. Sherman SI. Thyroid Carcinoma. Lancet (Lond Engl) (2003) 361(9356):50111. doi: 10.1016/s0140-6736(03)12488-9

5. Cabanillas ME, McFadden DG, Durante C. Thyroid Cancer. Lancet (Lond Engl) (2016) 388(10061):2783-95. doi: 10.1016/s0140-6736(16)30172-6

6. Adam MA, Pura J, Gu L, Dinan MA, Tyler DS, Reed SD, et al. Extent of Surgery for Papillary Thyroid Cancer Is Not Associated With Survival: An Analysis of 61,775 Patients. Ann Surg (2014) 260(4):601-5. doi: 10.1097/ sla.0000000000000925

7. Kuba S, Yamanouchi K, Hayashida N, Maeda S, Adachi T, Sakimura C, et al. Total Thyroidectomy Versus Thyroid Lobectomy for Papillary Thyroid Cancer: Comparative Analysis After Propensity Score Matching: A Multicenter Study. Int J Surg (Lond Engl) (2017) 38:143-8. doi: 10.1016/ j.ijsu.2016.09.083

8. Kim MJ, Lee MC, Lee GH, Choi HS, Cho SW, Kim SJ, et al. Extent of Surgery did Not Affect Recurrence During 7-Years Follow-Up in Papillary Thyroid

\section{DATA AVAILABILITY STATEMENT}

The original contributions presented in the study are included in the article/supplementary material. Further inquiries can be directed to the corresponding author.

\section{ETHICS STATEMENT}

Written informed consent was obtained from the individual(s) for the publication of any potentially identifiable images or data included in this article.

\section{AUTHOR CONTRIBUTIONS}

JL, JZ, SL, and HW conceived and designed the study. TM and HW conducted data collection and analyzed the data. TM wrote the manuscript. JL and TM revised the manuscript. All authors contributed to the article and approved the submitted version.

\section{FUNDING}

This research was supported by the special fund for deepunderground medical research (Grant No YB2018002) and 1.3.5 project for disciplines of excellence (Grant No.ZYJC18016 and ZYJC21048) provided by West China Hospital, Sichuan University, as well as the Sichuan International Technological Innovation Cooperation Project (Grant No.2018HH0159), National Natural Science Foundation of China (Grant No.51822403 and 81800892) and the research fund of Health commission of Sichuan province (Grant No.20PJ029).
Cancer Sized 1-4 Cm: Preliminary Results. Clin Endocrinol (2017) 87(1):80-6. doi: 10.1111/cen.13336

9. Vaisman F, Shaha A, Fish S, Michael Tuttle R. Initial Therapy With Either Thyroid Lobectomy or Total Thyroidectomy Without Radioactive Iodine Remnant Ablation Is Associated With Very Low Rates of Structural Disease Recurrence in Properly Selected Patients With Differentiated Thyroid Cancer. Clin Endocrinol (2011) 75(1):112-9. doi: 10.1111/j.1365-2265.2011.04002.x

10. Filetti S, Durante C, Hartl D, Leboulleux S, Locati LD, Newbold K, et al. Thyroid Cancer: ESMO Clinical Practice Guidelines for Diagnosis, Treatment and FollowUp†. Ann Oncol (2019) 30(12):1856-83. doi: 10.1093/annonc/mdz400

11. Haugen BR, Alexander EK, Bible KC, Doherty GM, Mandel SJ, Nikiforov YE, et al. 2015 American Thyroid Association Management Guidelines for Adult Patients With Thyroid Nodules and Differentiated Thyroid Cancer: The American Thyroid Association Guidelines Task Force on Thyroid Nodules and Differentiated Thyroid Cancer. Thyroid (2016) 26(1):1-133. doi: 10.1089/ thy. 2015.0020

12. NCCN Clinical Practice Guidelines in Oncology (NCCN Guidelines) Thyroid Carcinoma Version 2 (2020). Available at: https://www.nccn.org/ professionals/physician_gls/pdf/thyroid.pdf (Accessed October 15, 2020).

13. Siegel RL, Miller KD, Jemal A. Cancer Statistics, 2020. CA: Cancer J Clin (2020) 70(1):7-30. doi: 10.3322/caac.21590

14. Kitahara CM, Sosa JA. The Changing Incidence of Thyroid Cancer. Nat Rev Endocrinol (2016) 12(11):646-53. doi: 10.1038/nrendo.2016.110 
15. Ritter A, Bachar G, Hirsch D, Benbassat C, Katz O, Kochen N, et al. Natural History of Contralateral Nodules After Lobectomy in Patients With Papillary Thyroid Carcinoma. J Clin Endocrinol Metab (2018) 103(2):407-14. doi: 10.1210/jc.2017-01616

16. Ibrahim B, Forest VI, Hier M, Mlynarek AM, Caglar D, Payne RJ. Completion Thyroidectomy: Predicting Bilateral Disease. J Otolaryngol - Head Neck Surg = Le J D'oto-Rhino-Laryngologie Chirurgie Cervico-faciale (2015) 44(1):23. doi: 10.1186/s40463-015-0076-4

17. Wu ZG, Yan XQ, Su RS, Ma ZS, Xie BJ, Cao FL. How Many Contralateral Carcinomas in Patients With Unilateral Papillary Thyroid Microcarcinoma Are Preoperatively Misdiagnosed as Benign? World J Surg (2017) 41(1):12935. doi: 10.1007/s00268-016-3701-0

18. van Gerwen M, Alsen M, Lee E, Sinclair C, Genden E, Taioli E. RecurrenceFree Survival After Total Thyroidectomy and Lobectomy in Patients With Papillary Thyroid Microcarcinoma. J Endocrinol Invest (2021) 44(4):725-34. doi: 10.1007/s40618-020-01342-1

19. Amin MB, Edge S, Greene F, Byrd DR, Brookland RK, Washington MK, et al. AJCC Cancer Staging Manual. 8th ed. New York, NY: Springer (2017).

20. Carling T, Udelsman R. Thyroid Cancer. Annu Rev Med (2014) 65:125-37. doi: 10.1146/annurev-med-061512-105739

21. Davies L. Overdiagnosis of Thyroid Cancer. BMJ (Clin Res Ed) (2016) 355: i6312. doi: 10.1136/bmj.i6312

22. Welch HG, Doherty GM. Saving Thyroids - Overtreatment of Small Papillary Cancers. N Engl J Med (2018) 379(4):310-2. doi: 10.1056/NEJMp1804426

23. James BC, Timsina L, Graham R, Angelos P, Haggstrom DA. Changes in Total Thyroidectomy Versus Thyroid Lobectomy for Papillary Thyroid Cancer During the Past 15 Years. Surgery (2019) 166(1):41-7. doi: 10.1016/j.surg.2019.01.007

24. Mendelsohn AH, Elashoff DA, Abemayor E, St John MA. Surgery for Papillary Thyroid Carcinoma: Is Lobectomy Enough? Arch Otolaryngol-Head Neck Surg (2010) 136(11):1055-61. doi: 10.1001/archoto.2010.181

25. Hauch A, Al-Qurayshi Z, Randolph G, Kandil E. Total Thyroidectomy Is Associated With Increased Risk of Complications for Low- and High-Volume Surgeons. Ann Surg Oncol (2014) 21(12):3844-52. doi: 10.1245/s10434-0143846-8

26. Kandil E, Krishnan B, Noureldine SI, Yao L, Tufano RP. Hemithyroidectomy: A Meta-Analysis of Postoperative Need for Hormone Replacement and Complications. ORL J Otorhinolaryngol Rel Spec (2013) 75(1):6-17. doi: $10.1159 / 000345498$

27. Mizuno K, Takeuchi M, Kanazawa Y, Kitamura M, Ide K, Omori K, et al. Recurrent Laryngeal Nerve Paralysis After Thyroid Cancer Surgery and Intraoperative Nerve Monitoring. Laryngoscope (2019) 129(8):1954-60. doi: 10.1002/lary.27698

28. Hartl DM, Guerlain J, Breuskin I, Hadoux J, Baudin E, Al Ghuzlan A, et al. Thyroid Lobectomy for Low to Intermediate Risk Differentiated Thyroid Cancer. Cancers (2020) 12(11):3282. doi: 10.3390/cancers 12113282

29. Kletzien H, Macdonald CL, Orne J, Francis DO, Leverson G, Wendt E, et al. Comparison Between Patient-Perceived Voice Changes and Quantitative Voice Measures in the First Postoperative Year After Thyroidectomy: A Secondary Analysis of a Randomized Clinical Trial. JAMA OtolaryngolHead Neck Surg (2018) 144(11):995-1003. doi: 10.1001/jamaoto.2018.0309

30. Borel F, Tresallet C, Hamy A, Mathonnet M, Lifante JC, Brunaud L, et al. SelfAssessment of Voice Outcomes After Total Thyroidectomy Using the Voice Handicap Index Questionnaire: Results of a Prospective Multicenter Study. Surgery (2020) 167(1):129-36. doi: 10.1016/j.surg.2019.05.090

31. Falch C, Hornig J, Senne M, Braun M, Konigsrainer A, Kirschniak A, et al. Factors Predicting Hypocalcemia After Total Thyroidectomy - A Retrospective Cohort Analysis. Int J Surg (Lond Engl) (2018) 55:46-50. doi: 10.1016/j.ijsu.2018.05.014

32. Herranz González-Botas J, Lourido Piedrahita D. Hypocalcaemia After Total Thyroidectomy: Incidence, Control and Treatment. Acta Otorrinolaringol Espanola (2013) 64(2):102-7. doi: 10.1016/j.otorri.2012.09.001
33. Lale A, Öz B, Akcan AC, Sözüer EM, Arıkan TB, Gök M. Determination of Risk Factors Causing Hypocalcaemia After Thyroid Surgery. Asian J Surg (2019) 42(9):883-9. doi: 10.1016/j.asjsur.2018.12.009

34. Krekeler BN, Wendt E, Macdonald C, Orne J, Francis DO, Sippel R, et al. Patient-Reported Dysphagia After Thyroidectomy: A Qualitative Study. JAMA Otolaryngol-Head Neck Surg (2018) 144(4):342-8. doi: 10.1001/ jamaoto.2017.3378

35. Pereira JA, Girvent M, Sancho JJ, Parada C, Sitges-Serra A. Prevalence of Long-Term Upper Aerodigestive Symptoms After Uncomplicated Bilateral Thyroidectomy. Surgery (2003) 133(3):318-22. doi: 10.1067/msy.2003.58

36. Harries V, Wang LY, McGill M, Xu B, Tuttle RM, Wong RJ, et al. Should Multifocality Be an Indication for Completion Thyroidectomy in Papillary Thyroid Carcinoma? Surgery (2020) 167(1):10-7. doi: 10.1016/j.surg. 2019.03.031

37. Verloop H, Louwerens M, Schoones JW, Kievit J, Smit JW, Dekkers OM. Risk of Hypothyroidism Following Hemithyroidectomy: Systematic Review and Meta-Analysis of Prognostic Studies. J Clin Endocrinol Metab (2012) 97 (7):2243-55. doi: 10.1210/jc.2012-1063

38. Grani G, Ramundo V, Verrienti A, Sponziello M, Durante C. Thyroid Hormone Therapy in Differentiated Thyroid Cancer. Endocrine (2019) 66 (1):43-50. doi: 10.1007/s12020-019-02051-3

39. Kim SY, Kim HJ, Kim SM, Chang H, Lee YS, Chang HS, et al. Thyroid Hormone Supplementation Therapy for Differentiated Thyroid Cancer After Lobectomy: 5 Years of Follow-Up. Front Endocrinol (2020) 11:520. doi: $10.3389 /$ fendo.2020.00520

40. Lee YM, Jeon MJ, Kim WW, Sung TY, Chung KW, Shong YK, et al. Optimal Thyrotropin Suppression Therapy in Low-Risk Thyroid Cancer Patients After Lobectomy. J Clin Med (2019) 8(9):1279. doi: 10.3390/jcm8091279

41. Sun C, Wang Q, Guo Q, Chang Q, Liang X, Wang D, et al. Analysis of Patterns and Risk Factors Regarding the Onset of Nodules in the Contralateral Thyroid Lobe During Follow-Up Ultrasonography Among Patients After Unilateral Lobectomy for the Treatment of Papillary Thyroid Carcinoma. Head Neck (2020) 42(5):819-27. doi: 10.1002/hed.26056

42. Ibrahimpasic T, Nixon IJ, Palmer FL, Whitcher MM, Tuttle RM, Shaha A, et al. Undetectable Thyroglobulin After Total Thyroidectomy in Patients With Low- and Intermediate-Risk Papillary Thyroid Cancer-Is There a Need for Radioactive Iodine Therapy? Surgery (2012) 152(6):1096-105. doi: 10.1016/ j.surg.2012.08.034

43. Orosco RK, Hussain T, Noel JE, Chang DC, Dosiou C, Mittra E, et al. Radioactive Iodine in Differentiated Thyroid Cancer: A National Database Perspective. Endocrine-related Cancer (2019) 26(10):795-802. doi: 10.1530/erc-19-0292

44. Mazzaferri EL, Jhiang SM. Long-Term Impact of Initial Surgical and Medical Therapy on Papillary and Follicular Thyroid Cancer. Am J Med (1994) 97 (5):418-28. doi: 10.1016/0002-9343(94)90321-2

Conflict of Interest: The authors declare that the research was conducted in the absence of any commercial or financial relationships that could be construed as a potential conflict of interest.

Publisher's Note: All claims expressed in this article are solely those of the authors and do not necessarily represent those of their affiliated organizations, or those of the publisher, the editors and the reviewers. Any product that may be evaluated in this article, or claim that may be made by its manufacturer, is not guaranteed or endorsed by the publisher.

Copyright (c) 2021 Ma, Wang, Liu, Zou and Liu. This is an open-access article distributed under the terms of the Creative Commons Attribution License (CC BY). The use, distribution or reproduction in other forums is permitted, provided the original author(s) and the copyright owner(s) are credited and that the original publication in this journal is cited, in accordance with accepted academic practice. No use, distribution or reproduction is permitted which does not comply with these terms. 